\title{
FRACTAL MAPS DEPENDENCE ON SAR LOOK ANGLE
}

\author{
Gerardo Di Martino $^{l}$, Antonio Iodice ${ }^{1}$, Daniele Riccio ${ }^{l}$, Giuseppe Ruello ${ }^{1}$, Ivana Zinno ${ }^{2}$ \\ ${ }^{1}$ Università di Napoli Federico II, Via Claudio 21, 80125, Napoli, Italy \\ \{gerardo.dimartino,iodice,daniele.riccio,ruello\}@unina.it \\ ${ }^{2}$ Istituto per il Rilevamento Elettromagnetico dell'Ambiente (IREA), \\ Italian National Research Council (CNR), 80124, Napoli, Italy
}

\begin{abstract}
In this paper the application of an innovative SAR image processing developed by the authors - that provides a map of the point by point fractal dimension of a single (amplitude) SAR image - to a data-set of COSMO-SkyMed images, with look angles varying from $20^{\circ}$ to $45^{\circ}$, is presented. The main objective is the analysis of the fractal behavior of natural and urban scenarios, with particular evidence to the fact that fractal dimension estimation, in the case of SAR images of natural areas, turns out to be independent of the sensor viewing geometry, while for urban areas it is strongly dependent on look angle variations.
\end{abstract}

Index Terms - SAR, fractals, spectral analysis

\section{INTRODUCTION}

Nowadays, the necessity of developing tools for the interpretation and the information extraction from high resolution SAR data is a question of great relevance, since there is a strong need of providing an application counterpart to the huge amount of SAR images currently available. Hence, an innovative SAR image processing has been developed by the authors: working on a single (amplitude) SAR image, it provides the map of the point by point fractal dimension of the scene observed by the sensor [1], [2]. The fractal dimension is a significant parameter in describing natural surfaces' roughness and properties: as a matter of fact, it allows us to distinguish areas different from a geomorphologic point of view and to recognize geodynamic processes accountable for natural structures formation [3]-[5]. Furthermore, the fractal dimension retrieving turned out to be a very efficient instrument in differentiating automatically urban objects from natural ones: all natural surfaces hold a fractal dimension included in the interval ]2,3[, while man-made structures show a fractal dimension outside this interval [6].

In this paper the fractal maps generated by this innovative SAR image fractal processing applied to a set of SAR images acquired by the same sensor and relevant to the same area, but with different look angles, are analyzed and compared for the first time. The SAR data used for the fractal analysis are a set of COSMO-SkyMed stripmap images, with $3 \mathrm{~m} \times 3 \mathrm{~m}$ resolution, relevant to the area of Naples and surroundings and including both urban areas (the business district of Naples) and natural ones (the SommaVesuvius volcanic complex), with a look angle varying from $20^{\circ}$ to $45^{\circ}$. As a matter of fact, fractal and non fractal areas present a very different behavior, in terms of the retrieved fractal dimension, when some sensor parameters, and so the SAR image properties, change. The fractal dimension extraction, when dealing with natural areas, is fundamentally independent of the acquisition look angle; on the contrary, all the features of SAR images due to multiple scattering contributions from urban structures, generate differences in the fractal maps obtained starting from SAR images relevant to different SAR sensor look angles. Using the fractal dimension as a discriminator, the analysis of such differences, carried out comparing the statistical behaviors of the fractal maps estimated starting from urban or natural areas, is investigated in this paper. In order to analyze fractal maps relevant to SAR images of the same areas but acquired with different look angles, all the fractal maps have been firstly geocoded. Significant considerations concerning the provided fractal maps behavior are presented as well.

\section{FRACTAL DIMENSION ESTIMATION}

The fractal dimension retrieving is performed through a spectral analysis of the amplitude SAR image, according to the theoretical analysis presented by the authors in [1]. In particular, dealing with power-law spectra, an appropriate spectral estimator, that minimizes high variance and leakage problems, is used: the Capon estimator [7].

In order to obtain a point-by-point map of the fractal dimension the spectral estimation is performed using a window sliding over the whole SAR image: the window encloses small portions of the image that are supposed to share the same fractal parameters and a unique value of fractal dimension is assigned to the center pixel of each window. The dimension of the sliding window results from a trade-off between the accuracy of the estimation and the 


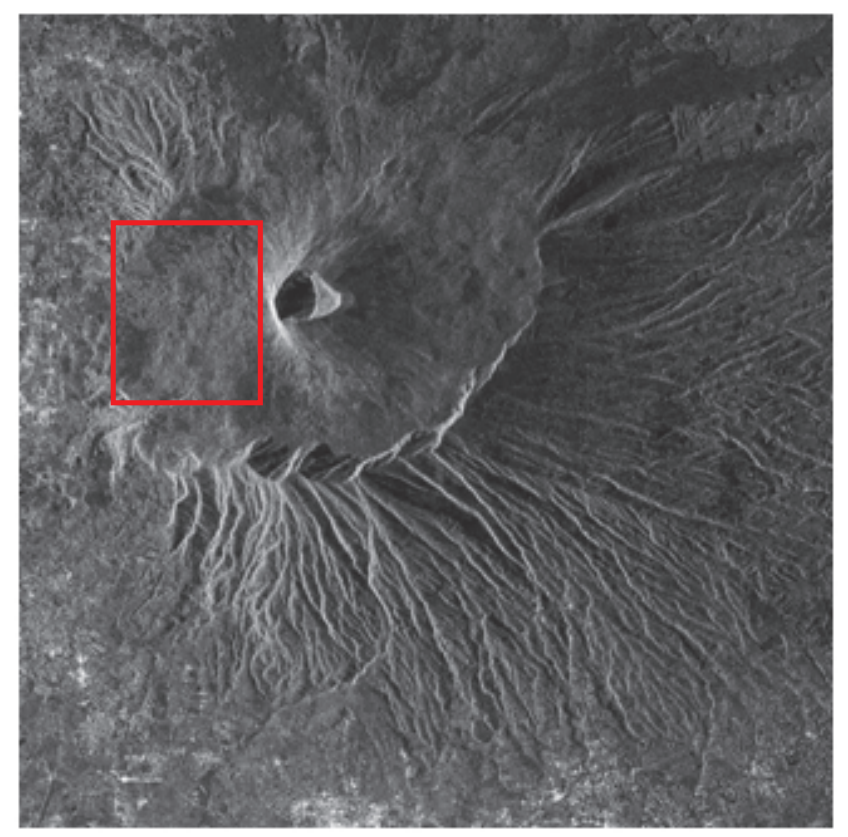

Fig. 1: COSMO-SkyMed stripmap SAR image (resolution 3mx3m) of the Somma-Vesuvius volcanic complex (Naples, Italy). The image dimensions are 4000x4000 pixels.

resolution required for the generated fractal maps. It is evident that an appropriate choice of the sliding window dimension is of key importance whenever the analysis and interpretation of the fractal maps is in order. In fact, accuracy and resolution of the obtained maps can play different roles according to the particular parameter of interest.

In this paper the main objective is the comparison between the behaviors of fractal maps obtained starting from SAR images acquired with different look angles, in the case either of urban or natural areas. Concerning this, the fractal maps are generated using a sliding window of $51 \times 51$ pixels, that is a dimension that assures a good trade-off between resolution and accuracy, with an intermediate filtering (the Capon filtering length is set to $0.3 * N, N$ being the number of samples) and with a standard anti-aliasing filtering.

\section{ANALYSIS OF THE FRACTAL MAPS}

In order to perform the fractal analysis described in the preceding sections, a set of COSMO-SkyMed stripmap images, with $3 \mathrm{mx} 3 \mathrm{~m}$ resolution, relevant to the area of Naples and surroundings and including both urban areas (the business district of Naples) and natural ones (the SommaVesuvius volcanic complex), acquired respectively with look angles of $22^{\circ}, 31^{\circ}$ and $44^{\circ}$, are used.

Only for the sake of brevity, in figures 1, 2 only the cuts of the $31^{\circ} \mathrm{SAR}$ image relevant to the Vesuvius area and of a part of the urban area are shown. The same geographic areas have been cut out from the SAR images acquired with look angles equal to $22^{\circ}$ and $44^{\circ}$.

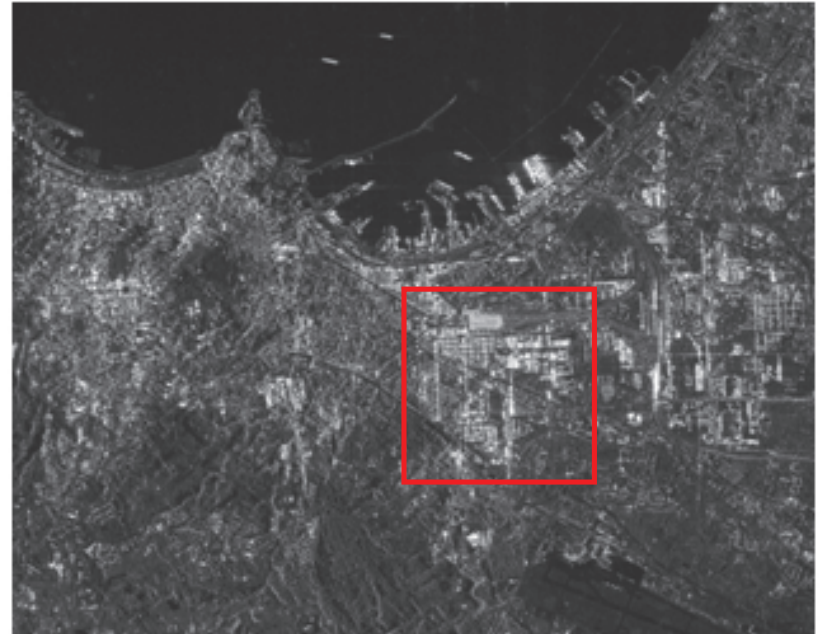

Fig. 2: COSMO-SkyMed stripmap SAR image (resolution 3mx3m) of an urban area of Naples (Italy). The image dimensions are $4442 \times 3902$ pixels.

Table I

\begin{tabular}{|c|c|c|c|c|}
\hline & $\mathrm{D}_{\min }$ & $\mathrm{D}_{\max }$ & $\mathrm{D}_{\text {mean }}$ & Stddev \\
\hline Fig. 3 $\left(22^{\circ}\right)$ & 1.286 & 2.737 & 2.241 & 0.095 \\
\hline Fig. 4 $\left(31^{\circ}\right)$ & 1.364 & 2.717 & 2.230 & 0.098 \\
\hline Fig. 5 $\left(44^{\circ}\right)$ & 1.252 & 2.659 & 2.222 & 0.104 \\
\hline
\end{tabular}

Table II

\begin{tabular}{|c|c|c|c|c|}
\hline & $\mathrm{D}_{\min }$ & $\mathrm{D}_{\max }$ & $\mathrm{D}_{\text {mean }}$ & Stddev \\
\hline Fig. 6 $\left(22^{\circ}\right)$ & 1.063 & 3.738 & 2.233 & 0.155 \\
\hline Fig. 7 $\left(31^{\circ}\right)$ & 1.013 & 4.316 & 2.211 & 0.171 \\
\hline Fig. 8 $\left(44^{\circ}\right)$ & 1.007 & 4.065 & 2.183 & 0.188 \\
\hline
\end{tabular}

In Fig. 1-3 and in Fig. 4-6, the fractal maps relevant to the geographic areas represented in Fig. 1 and 2, obtained respectively from the $22^{\circ}, 31^{\circ}$ and $44^{\circ}$ SAR images are presented. In Tables I and II the statistics of the fractal maps of Fig. 1-3 and Fig 4-6 are reported.

Recalling that the fractality interval for natural scenes is ]2,3[, and in particular natural surfaces are expected to hold a fractal persistent behavior [4,5], i.e. $D \in] 2,2.5]$, some initial considerations about the statistics of the provided fractal maps can be drawn. First of all, the minimum and maximum values assumed by the fractal dimension are significantly different in the natural areas case, where the maximum of $D$ never reaches the superior limit of the range of fractality ]2,3[ and in the urban area case where - on the contrary - the fractal dimension assumes values much greater than 3. The same behavior, also if in a less evident way, is assumed by the minimum values of the estimated $D$, that, in the natural areas case are globally greater than those of the urban areas. The fractal dimension estimation provides values lower than 2 (that in the fractal maps of Fig. 3-8 correspond to the darker level of grey), and so below the range of fractality, when an urban object is present in the window used for the estimation [6]. As a matter of fact, in 


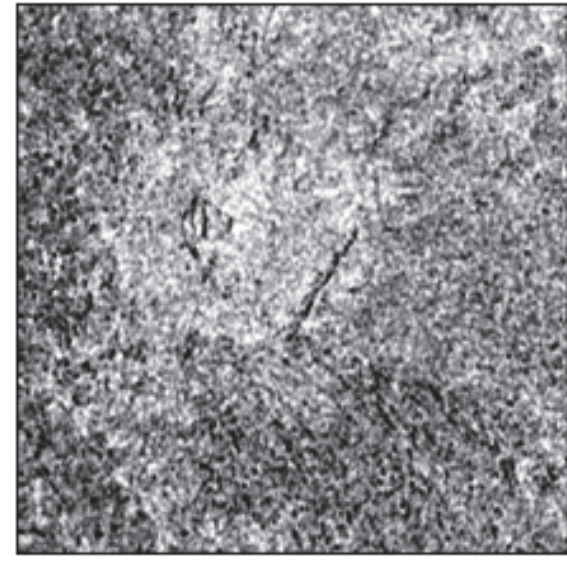

Fig. 3: Fractal map relevant to the area represented in Fig. 1 obtained from the SAR image with $22^{\circ}$ look angle

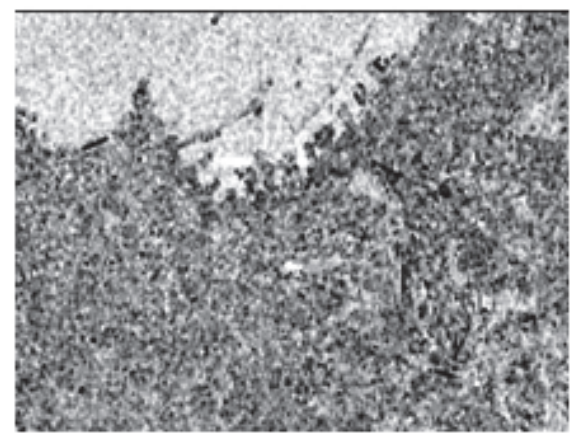

Fig. 6: Fractal map relevant to the area represented in Fig. 2 obtained from the SAR image with $22^{\circ}$ look angle

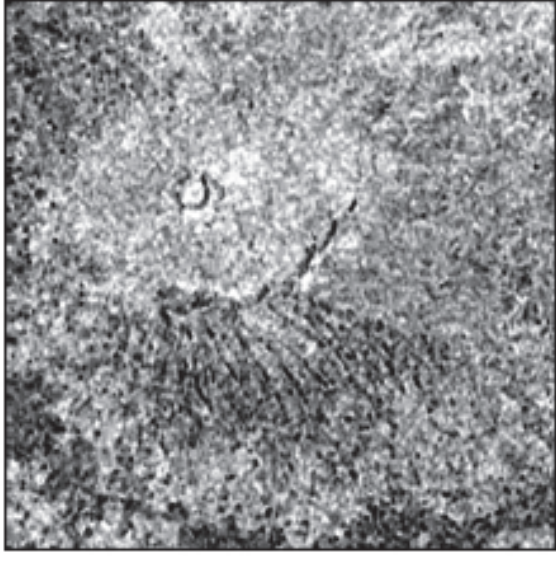

Fig. 4: Fractal map relevant to the area represented in Fig. 1 obtained from the SAR image with $31^{\circ}$ look angle

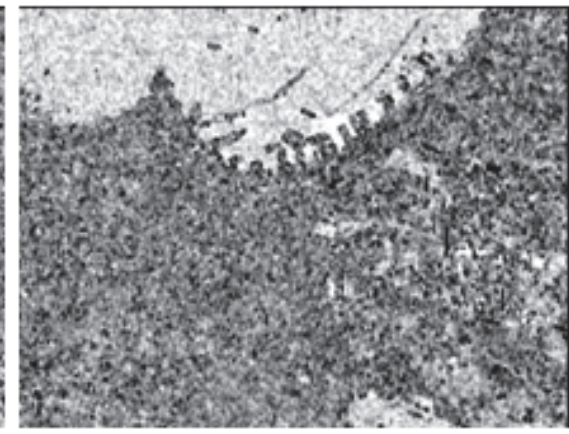

Fig. 7: Fractal map relevant to the area represented in Fig. 2 obtained from the SAR image with $22^{\circ}$ look angle

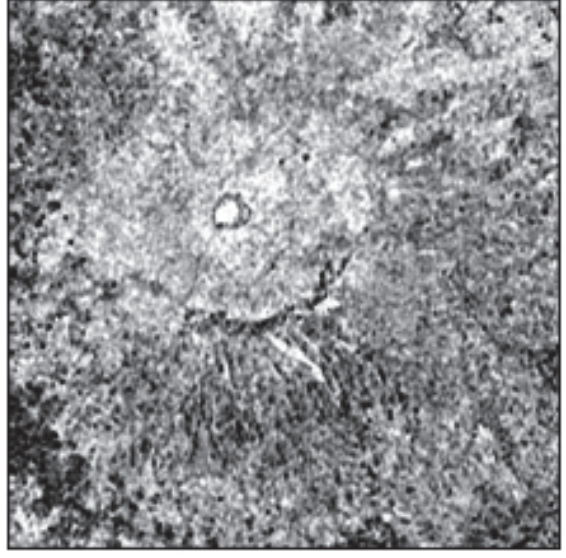

Fig. 5: Fractal map relevant to the area represented in Fig. 1 obtained from the SAR image with $44^{\circ}$ look angle

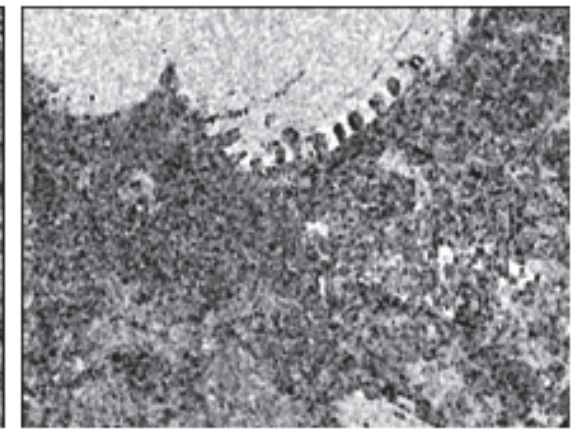

Fig. 8: Fractal map relevant to the area represented in Fig. 2 obtained from the SAR image with $22^{\circ}$ look angle the case of a SAR image of natural surfaces described through a fractal model, the image spectrum holds a powerlaw behavior whose expression depends on the fractal dimension of the imaged scene. The presence of man-made structures gives rise, in a SAR image, to particularly brilliant points and multiple reflection lines that strongly modify the image spectrum evaluated around these points, leading to a fractal dimension estimate that significantly diverges from the fractal case. Note that also the layover zones are recognized as non-fractal with a $D$ lower than 2 .

The values of $D$ that exceed 3 (that in the fractal maps of Fig. 3-8 correspond to the brighter level of grey), instead, are due either to shadowing phenomena or to the SAR image features, produced by the SAR point spread function, that appear when there is a dihedral object that acts as a corner reflector. In this case, in the spectrum estimation window, there is an entire bright line in the range direction, thus leading estimated $D$ values greater than 3 .

In order to perform a more specific analysis regarding the SAR images acquired with different look angles, the fractal maps shown in Fig. 3-8 have been geocoded so that corresponding areas could be exactly compared.

Concerning the Vesuvius fractal maps, to consider a solely natural area, the zone of the volcano's foreslope has been cut out from the three $\left(22^{\circ}, 31^{\circ}, 44^{\circ}\right)$ geocoded fractal maps. Similarly, from the three geocoded fractal maps of the urban area, the zone of the business district of Naples has been cut out. Such areas are indicated in the red boxes in Fig. 1, 2.

In Fig. 9, 10 and in Tables III, IV the histograms and the statistics of the geocoded fractal maps relevant to the above described cuts are reported. The histograms are all normalized to the total number of pixels and have a binsize equal to 0.01 .

As the figures clearly show the behaviors of the estimated fractal dimension maps for SAR images of natural and urban areas, depending on diverse SAR look angles, are very different. In the first case the fractal dimension estimation turns out to be absolutely independent on the SAR view geometry: statistics presented in Table 3 are fundamentally equivalent as the look angle varies as well as 


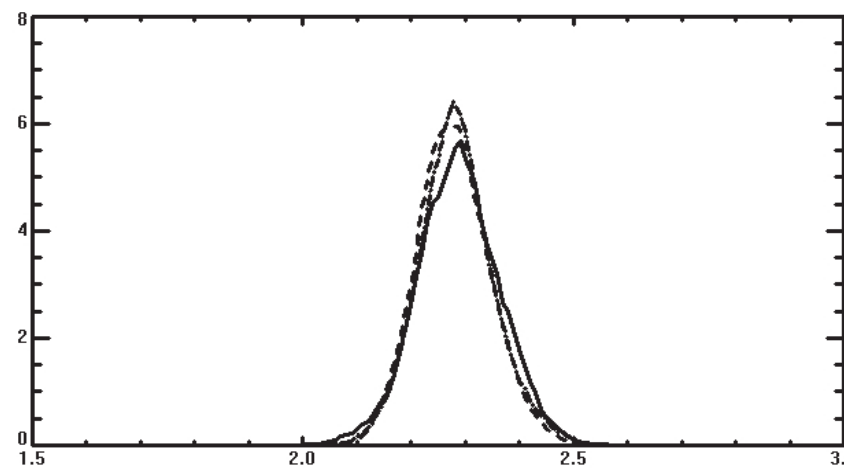

Fig. 9: Superimposed histogram (binsize $=0.01$ ) relevant to:

- fractal map of the Vesuvius' foreslope area of $22^{\circ}$ SAR image - continuous line

- fractal map of the Vesuvius' foreslope area of $31^{\circ}$ SAR image - dash line

- fractal map of the Vesuvius' foreslope area of $44^{\circ}$ SAR image - dash/dotted line

Table III

\begin{tabular}{|c|c|c|c|c|}
\hline & $\mathrm{D}_{\min }$ & $\mathrm{D}_{\max }$ & $\mathrm{D}_{\text {mean }}$ & Stddev \\
\hline $\begin{array}{c}\text { Fig. 9 } \\
\text { (continuous line) }\end{array}$ & 1.979 & 2.582 & 2.292 & 0.076 \\
\hline $\begin{array}{c}\text { Fig. 9 } \\
\text { (dash line) }\end{array}$ & 2.038 & 2.601 & 2.283 & 0.067 \\
\hline $\begin{array}{c}\text { Fig. 9 } \\
\text { (dash-dotted line) }\end{array}$ & 2.009 & 2.573 & 2.288 & 0.069 \\
\hline
\end{tabular}

Table IV

\begin{tabular}{|c|c|c|c|c|}
\hline & $\mathrm{D}_{\min }$ & $\mathrm{D}_{\max }$ & $\mathrm{D}_{\text {mean }}$ & Stddev \\
\hline $\begin{array}{c}\text { Fig. 10 } \\
\text { (continuous line) }\end{array}$ & 1.167 & 3.236 & 2.199 & 0.138 \\
\hline $\begin{array}{c}\text { Fig.10 } \\
\text { (dash line) }\end{array}$ & 1.037 & 3.354 & 2.162 & 0.145 \\
\hline $\begin{array}{c}\text { Fig. 10 } \\
\text { (dash-dotted line) }\end{array}$ & 1.244 & 3.315 & 2.138 & 0.160 \\
\hline
\end{tabular}

the histograms' shapes. Minimal differences can be adduced to the estimation error.

It is worth noting that, being in this case the considered cut relevant to a completely natural area (in absence of buildings or of any man-made object), the estimated fractal dimension keeps firmly in the fractality range ]2,3[. On the contrary, the histograms and the statistics of the fractal maps relevant to the urban area present a clear dependence on the SAR look angle. In particular the mean fractal dimension decreases as the look angle increases, and the histogram shape tends to drop and to widen out.

\section{CONCLUSIONS}

In this paper the application of an innovative SAR image post-processing developed by the authors - that provides a map of the point by point fractal dimension of a single (amplitude) SAR image - to a data-set of COSMOSkyMed

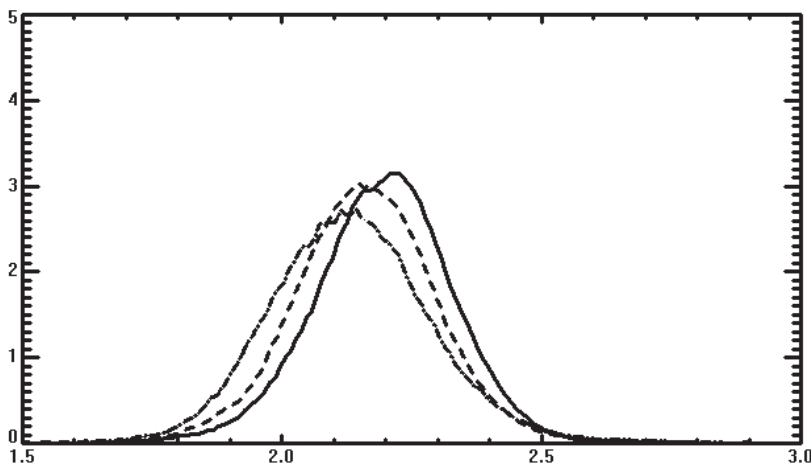

Fig. 10: Superimposed histogram (binsize $=0.01$ ) relevant to:

- fractal map of the Naples' business district area of $22^{\circ}$ SAR image - continuous line

- fractal map of the Naples' business district area of $31^{\circ}$ SAR image - dash line

- fractal map of the Naples' business district area of $44^{\circ}$ SAR image - dash/dotted line

stripmap images of Naples (Italy), with look angles varying from $20^{\circ}$ to $45^{\circ}$, is presented. The considered SAR data set is relevant to an area with both an urban centre (the business district of Naples) and a significant natural scenario (the Somma-Vesuvius volcanic complex), thus giving the opportunity to analyze the fractal behavior of natural and urban scenarios. It has been shown that while the fractal dimension estimated from a SAR image relevant to natural areas turns out to be independent of the sensor viewing geometry, this does not hold for the fractal dimension pertinent to urban areas where features owing to multiple scattering contributions are present and modify the fractal estimation depending on the SAR viewing geometry.

\section{REFERENCES}

[1] G. Di Martino, D. Riccio and I. Zinno, "SAR Imaging of Fractal Surfaces", IEEE Trans. Geosci. Remote Sens., vol.50, no.2, pp. 630-644, Feb. 2012.

[2] G. Di Martino, A. Iodice, D. Riccio, G. Ruello and I. Zinno, "Fractal Based Filtering of SAR Images", Proceedings IGARSS 2010, Honolulu (USA), July 2010, pp. 2984-2987

[3] B.B. Mandelbrot, The Fractal Geometry of Nature. New York: Freeman, 1983

[4] G. Franceschetti and D. Riccio, Scattering, Natural Surfaces and Fractals, Burlington, MA: Academic, 2007.

[5] Turcotte D.L. 1997 - Fractals and Chaos in Geology and Geophysics (s. e.) Cambridge University Press (pp:1-385)

[6] G. Di Martino, A. Iodice, D. Riccio, G. Ruello and I. Zinno, "Fractal Filtering Applied to SAR Images of Urban Areas", Proceedings of Joint Urban Remote Sensing Event 2011, Munich (Germany), April 2011, pp. 261-264

[7] T. Austin, A. W. England, G. H. Wakefield, "Special problems in the estimation of power-law spectra as applied to topographical modeling”, IEEE Trans. Geosci. Remote Sens., vol. 32, no. 4, pp. 928-939, July 1994. 